2021, T. 163, кH. $1 \quad$ ISSN 2541-7746 (Print)
C. $21-30$
ISSN 2500-2198 (Online)

ORIGINAL ARTICLE

UDC 535.23:621.378.825

doi: 10.26907/2541-7746.2021.1.21-30

\title{
ON SOLVING THE PROBLEM OF QUASI-TWO-DIMENSIONAL SUPERCRYSTAL NONLINEAR RESONANCE RESPONSE
}

\author{
E.V. Timoshchenko ${ }^{a}$, V.A. Yurevich \\ ${ }^{a}$ Mogilev State A. Kuleshov University, Mogilev, 212022 Republic of Belarus \\ ${ }^{b}$ Belarusian State University of Food and Chemical Technologies, \\ Mogilev, 212027 Republic of Belarus
}

\begin{abstract}
Taking into account the foundations of the generalized two-level scheme, an analytical solution to the problem of the evolution of superradiance in a quasi-two-dimensional supercrystal formed by quantum dots is obtained under homogeneous lasing field assumption in the resonant medium of the quasicrystal. The calculation was performed for the physical parameters of a semiconductor structure with quantum-well effects in the presence of resonant nonlinearity and intraband relaxation. We use the generalized two-level scheme, which allows us to take into account the self-modulating spectral broadening of the light field due to the absorption of radiation in quasi-resonant transitions in the quantum mechanical material equations, which are solved together with the field coupling equations. A relation is formulated that is analogous to the law of conservation of the polar angle of the Bloch vector for the more general case of interaction under consideration, in which, along with the phase nonlinearity of the response, the spread rate of active dipoles within the spectral line width is taken into account (i.e., the finiteness of the phase relaxation time of elementary emitters). The use of the Bloch vector formalism in this case makes it possible to obtain an analytical solution of the original modification of the nonlinear system of equations for the semiconductor supercrystals response variables and to calculate the shape of the superradiance pulses. The calculations predict the pronounced asymmetry of the pulses emitted by the semiconductor supercrystals. The calculated estimates of the time dynamics of the superradiance process, taking into account the nonlinearities typical for the resonant response, can be used in the development of methods for obtaining and profiling optical pulses in the sub-picosecond range of durations in modern compact nanophotonics devices.
\end{abstract}

Keywords: nonlinear dynamics, quantum dots supercrystals, resonance material response, dipole-dipole interaction, collective effects of radiation, Bloch vector formalism

\section{Introduction}

To date, artificial electromagnetic composite media with optical properties that are absent in their constituent raw materials have been actively developed. These previously unobvious optical or, in a wider frequency range, electromagnetic properties are due to the sub-wave size of elementary dipole emitters when they are densely packed in a medium and the resonant nature of the scattering of external electromagnetic radiation. It has been established that two-dimensional supercrystals from regularly located dipole centers (in particular quantum dots) are one example of the objects that can have their optical properties easily controlled by a change in the shape and chemical composition of the forming elements, as well as in their geometry [1]. Metamaterials 
also belong to a new class of substances that do not exist in nature [2]. The internal structure of many quasicrystals, including transition metal dichalcogenides (for example, $\mathrm{MoSe}_{2}, \mathrm{WSe}_{2}$ ) [3], supercrystals of semiconductor quantum dots [4, 5], phosphorenes [6], and organic polymers [7], can be represented by an ordered ensemble of quantum dots (meta-atoms, i.e., formations that exceed the average atomic size, for example, excitons) with discrete properties of the energy spectrum. Owing to the existence of additional free degrees in the scheme of resonant interaction with coherent radiation (for example, lattice geometry and the interaction of quantum dots represented by dipoles), supercrystals having a nanoscale thickness are characterized by unique transport and optical properties. A supercrystal is able to almost completely reflect the light field in a certain spectral range, presenting in this way an ideal frequency-critical mirror of nanometer thickness. In addition, reflectivity of such objects detects bistable behavior, i.e., it can be switched by a slight change in the intensity of the external signal from the final value to the mode of almost complete transparency [8]. The advantage of two-dimensional supercrystals is largely expressed by the fact that the vast majority of their materials are semiconductors, the energy structure of which can be easily controlled by external action, thereby making them highly promising for nanophotonic applications. Quantum transitions on excitonic level schemes are characterized by gigantic oscillator forces, which leads to a more powerful manifestation of nonlinear optical effects at moderate values of intensity of coherent radiation acting in a supercrystal medium. Therefore, studying the laws of nonlinear dynamics of the resonant response of supercrystals formed by quantum dots seems relevant and appropriate from a practical point of view.

\section{Description of the problem and basic equations}

In the present work, the problem of the evolution of stimulated emission in the structure of a low-dimensional supercrystal is posed, provided that the characteristic duration of the generated pulses is much shorter than the duration of the matter relaxation mechanisms.

Under this condition, the interaction of the medium and the light field is coherent (oscillations of the resonance polarization of the ensemble of active centers and the wave field generated by it are consistent), and one of the collective effects caused by this correlation is represented by superradiation (SR) [9]. The emission of a superradiance pulse by an ensemble of a large number $N$ of inverted active centers can develop over time of $\tau_{R}$, which is much shorter than the characteristic radiation time $T_{1}$ of an isolated atom.

The phenomenon of superradiation occurs as a result of spontaneous phase correlation (phase matching or phasing) of initially independent active centers forming a supercrystal. The appearance of phase correlation is explained by the following two factors: the interaction of dipoles through the electromagnetic field emitted by them and the nonlinearity of oscillatory electron motions inside active centers.

Semiconductor structures with quantum effects are used as inverse media; in the form of planar layers, these materials are used as active surface films capable of modulating resonant radiation. In semiconductor media, SR develops as a collective spontaneous recombination [10]. During the SR pulse formation, the phases of individual excitons represented by dipoles are spontaneously synchronized, as a result of which a collective dipole with a short radiative recombination time is formed within the supercrystal.

The influence of the dipole - dipole interaction and the reaction on the field of transitions close to the main one, as the population resonance changes, determines the shift of the center of the spectral gain line and the self-modulation frequency drift of the emitted field. Due to the relatively dense packing of active centers in the supercrystal structure and high values of dipole moments, these features inherent in resonance interaction can determine the self-dephasing of an ensemble of elementary emitters during SR pulse formation. 
In the present work, the influence of these factors of nonlinear phase dynamics on the process of superradiation in a quasi-two-dimensional supercrystal of quantum dots is taken into account. The phase effects determine the possibility of dynamic feedback occurrence. Therefore, as applied to such low-dimensional amplifying elements, it is necessary to speak about the lasing with the specificity of the SR process.

Quantum-sized structures possess the properties of materials formed by active centers, the reaction of which to the electromagnetic radiation field has a pronounced dipole character. Therefore, the two-level scheme concepts of the interaction of the matter with a resonant light field are applicable to their calculation analysis. An optical plane wave field in a supercrystal layer is naturally assumed to be homogeneous in the direction of its thickness.

In contrast to the already solved analytical problems on the SR subject, the solution along with the phase relaxation of elementary dipoles takes into account the polarizing effect on the matter dielectric susceptibility of the near fields of the dipoles in the framework of the representation of the acting field, including the local Lorentz correction. Such kind of nonlinear mechanism of interaction between the matter and the field, which leads to a low-inertial shift of the spectral line, is typical precisely for media with a relatively high density of active centers [11].

Generalization of the two-level scheme in the problem considered below consists in taking into account the effect on the polarizability of absorption in quasi-resonant transitions. This is usually expressed by the assumption of a difference (defect) in the polarizabilities of the dipole particles with values of $\alpha_{1}$ and $\alpha_{2}$ in the ground and excited states [12]. When particles are redistributed over the transition levels during stimulated emission, nonlinear resonant refraction variations are possible, which is also significant in semiconductor excitonic media.

The problem of energy exchange of a supercrystal medium and a light field during SR can be solved by assuming an ultrathin boundary layer of a resonant material, in essence, for a planar film radiating from a surface. In this case, instead of wave equations, the use of boundary electrodynamic conditions in the Maxwell equations written for the planewave field acting on atoms and reflected by the layer is allowed [13].

The light field acting on the active centers and the probability of resonant polarization are represented in the form of quasi-harmonic waves with a carrier frequency close to the frequency of the fundamental transition $\omega_{0}$, and relatively slow (slightly varying over the period of the light oscillation) complex amplitudes $E(t)$ and $i \rho(t)$, i.e., $E(t)=E(t) \exp (i \omega t), \rho(t)=i \rho(t) \exp (i \omega t)$.

Together with the relations for the light fields, it is customary to consider quantummechanical two-level density matrix equations for the probabilistic variables of the resonance polarization and the level of population difference.

In this case, the quantum-mechanical equations of motion can be reduced to the socalled optical Bloch equations [9] for the quasi-stationary polarization amplitude $\rho(t)$ and the population inversion variable $n(t)$, for which the relaxation dynamics is determined by the "slow" field strength and polarization amplitudes (their envelopes).

The derivation of analogues of this model of energy exchange of radiation with a resonantly polarizable thin layer under the condition of homogeneously broadening spectral line of absorption is sufficiently substantiated in the literature (see, for example, [14]). Therefore, we will preliminarily write the system of Maxwell-Bloch equations used below for the analysis of the quasicrystal resonance reaction to a light field in the form:

$$
\begin{aligned}
& E=t_{0} E_{i}-i \frac{\omega l}{\varepsilon_{0} c} P, \quad E_{r}=-r_{0} E_{i}-i \frac{\omega l}{\varepsilon_{0} c} P, \quad P=i \mu N \rho, \\
& \frac{d \rho}{d t}+\frac{1}{T_{2}} \rho+i\left(\omega_{0}-\omega\right) \rho=\frac{\mu}{\hbar} n E, \quad \frac{d n}{d t}+\frac{n-n_{0}}{T_{1}}=-\frac{\mu}{2 \hbar}\left(\rho^{*} E+\rho E^{*}\right) .
\end{aligned}
$$


In system (1), $E_{i}$ and $E_{r}$ are the amplitudes of the externally incident and reflected field strength, $t_{0}$ and $r_{0}$ are the Fresnel transmission and reflection coefficients of the quasicrystal surface, $\mu$ is the average dipole moment of the active centers, $N$ is their volume concentration in a layer of thickness $l, T_{2}$ is phase (transverse or intraband) relaxation time characterizing the spectral width of the gain line, $n_{0}$ is the initial inversion level. In addition to the Fresnel components that are included in the boundary condition expressions and are proportional to the amplitude of the macroscopic polarization $P$, are called superradiant. It is assumed that the dynamics of these nonlinear components can characterize the evolution of the coherent field interacting with such objects as quasi-two-dimensional supercrystals [15].

In evaluating the consequences of the dipole - dipole interaction, similarly to [14], we neglect the relatively static nonresonant contribution to the local field. Then the amplitude of the intensity of the field acting on the active centers is represented taking into account the local Lorentz correction in the expression: $E^{\prime}=E+\frac{P}{3} \epsilon_{0}$, which is further applied in the computational model (1). Using the generalized two-level scheme, within the framework of system (1), it is relatively easy to take into account the contribution to the resonant polarization of radiation from transitions adjacent to the resonance one. The value of the polarizability defect is used as an important characteristic of the polarizing effect of the field corresponding to quasi-resonant transitions $\Delta \alpha=\alpha_{2}-\alpha_{1}$ (difference in polarizabilities of active atoms at the levels of the main transition). The quantity $\Delta \alpha$ determines the velocity of the phase drift of the field (frequency "chirp") caused by nonlinear refraction. Based on the conclusions of [12], to take into account its consequences, instead of the polarization amplitude $P(t)=i \mu N \rho(t)$, we can use its value refined within the framework of the generalized two-level scheme, where the quasi-resonant term is highlighted:

$$
P(t)=N\left[i \mu \rho+2 \pi \Delta \alpha \varepsilon_{0}\left(n_{0}-n\right) E^{\prime}\right] .
$$

The influence of transitions adjacent to the resonance one is also related the quadratic Stark effect possibility [12]: in the approach of the generalized two-level scheme, the field-induced Stark shift of resonance levels is described by one of the nonlinear components of the phase detuning in the equation for polarization in the Bloch system [16].

Taking into account the Lorentzian correction and representation (2) similarly to [17] makes it possible to correspondingly modify system (1). When recording it, we neglect the longitudinal (interband) relaxation and normalize the variable field strengths by reducing them to quantities that have no dimension $\left(\mathbf{e}^{\prime}=\mu_{2} E^{\prime} / h, \mathbf{e}_{i}=\mu E_{i} / h\right)$ :

$$
\begin{aligned}
& \mathbf{e}^{\prime}=t_{0} \mathbf{e}_{i}+\frac{1}{\tau_{R}}\left[T_{2} \rho-i \frac{2 \pi \Delta \alpha \varepsilon_{0} \hbar}{\mu^{2}}\left(n_{0}-n\right) \mathbf{e}^{\prime}\right](1+i \gamma), \quad \mathbf{e}_{r}=\mathbf{e}-\mathbf{e}_{i}, \\
& T_{2} \frac{d \rho}{d t}-\left[1+i\left(\omega_{0}-\omega\right) T_{2}\right] \rho=n \mathbf{e}^{\prime}, \quad T_{2} \frac{d n}{d t}=-\frac{1}{2}\left(\rho^{*} \mathbf{e}^{\prime}+\rho \mathbf{e}^{\prime *}\right),
\end{aligned}
$$

where $\tau_{R}=\epsilon_{0} c \hbar / \mu^{2} \omega N l$ is superradiance time, $\gamma=c / 3 \omega l$ is normalization factor in the phase component arising when the local Lorentz correction is taken into account.

Superradiance arises as a result of inversion dumping when its high values are reached in the active medium; it can develop without a light field initiating from the outside, and at the start of the process the frequency of the acting field coincides with the central frequency of the spectral gain line. In system (3), then it is obviously necessary to set $\mathbf{e}_{i}(t) \equiv 0$ and $n_{0}=1$, as well as to consider the case of exact 
resonance $\omega=\omega_{0}$. The $\mathrm{SR}$ computation model is then presented in the following form:

$$
\begin{aligned}
& T_{2} \frac{d \rho}{d t}=\left\{\frac{\kappa n}{[1-\beta \gamma(1-n)]^{2}+\beta^{2}(1-n)^{2}}-1\right\} \rho+i \frac{\kappa n\left[\gamma-\beta\left(1+\gamma^{2}\right)(1-n)\right]}{[1-\beta \gamma(1-n)]^{2}+\beta^{2}(1-n)^{2}} \rho, \\
& T_{2} \frac{d n}{d t}=-\frac{\kappa|\rho|^{2}}{[1-\beta \gamma(1-n)]^{2}+\beta^{2}(1-n)^{2}}, \quad u(t)=\frac{\kappa^{2}|\rho|^{2}}{[1-\beta \gamma(1-n)]^{2}+\beta^{2}(1-n)^{2}},
\end{aligned}
$$

In system (4), it seems convenient to use the parameter of the layer unsaturated gain index (it is also called the parameter of the resonant nonlinearity of absorption (gain)); to estimate its value, the ratio $\kappa=T_{2} / \tau_{R}$ is considered correct [9]. A nonlinear refraction coefficient was also introduced: $\beta=2 \pi \Delta \alpha \omega N l / c$, which determines the rate of nonlinear frequency detuning and self-modulation change in SR dynamics. Note that in the equation for polarization in system (4) the imaginary component is specially highlighted, which describes the estimate of the magnitude and dynamics of the relative phase shift of the field and polarization. Its factors are the influence of the near-field dipoles and the presence of transitions adjacent to the ground, capable of responding to resonance radiation. Under the assumptions used, the SR power density $u(t)$ is determined by the superradiant component of the resonant polarization - it is proportional to the square of the polarization. In calculations based on (4), the SR power density $u(t)$ has no dimension - it is normalized by the value of $\left(h / \mu T_{2}\right)^{2}$.

\section{Solution of the problem for the coordinates of the Bloch vector}

The dynamics of the response components of the quasicrystal medium determines the amplification process when a certain threshold inversion is reached. It can be assumed that at the initial stage of SR, the dipole ensemble representing the quasicrystal should be maximally inverted. For example, it should be accepted, in accordance with tradition [9], that at the initial moment $n(t=0)=1$, while there are no correlations in the ensemble: $\rho(t=0)=0$. It is now easy to determine that solutions (4) for $\rho$ and $n$ satisfy the following relation:

$$
|\rho|^{2}+n^{2}=1-\frac{2}{\kappa}(1-n)+\frac{2}{\kappa} \beta(1-n)^{2}\left[\gamma-\frac{\beta}{3}\left(1+\gamma^{2}\right)(1-n)\right] .
$$

In the literature devoted to the theoretical analysis of collective effects, it is considered convenient to use the Bloch vector formalism. Variables of equations similar to the computational model (4) are then considered in the form of coordinates (components) of the Bloch vector: $X=\operatorname{Re} \rho, Y=\operatorname{Im} \rho, Z=n$. In the absence of effects causing a nonlinear frequency detuning (the "classical" case is for an extended medium), the relation is formulated $|\rho|^{2}+n^{2}=1$, known as the law of conservation of the polar angle of the Bloch vector [9]. Note that expression (5) represents, in essence, an analogue of the conservation of the polar angle of the Bloch vector for a more general case of interaction. Along with phase nonlinearity, the formulation of (5) takes into account the rate of dispersion of dipoles within the width of the spectral line (the finiteness of the time of phase relaxation of dipoles). The generality of formulation (5), however, is limited by the reasonable assumption of a inhomogeneous field in a quasi-two-dimensional quasicrystal.

\section{Calculation results}

In the choice of parameters determining the magnitudes of the coefficients (4) for semiconductor quantum-dimensional structures, we mainly oriented ourselves on data known, for example, from such papers as $[5,10]$. The approximate scale of the phenomena corresponded to the intensity of the order $(1 \ldots 5) \cdot 10^{6} \mathrm{Wt} / \mathrm{cm}^{2}$, concentration 


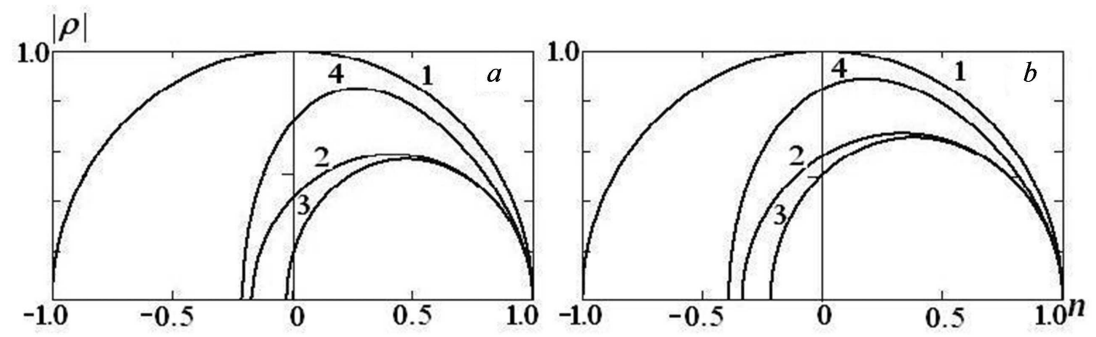

Fig. 1. The shape of the parts of the phase trajectories in the $(|\rho|, n)$ plane (curves 1 correspond to the "classical" case of an extended medium): $\kappa=2.4(a), 3.0(b), \beta=0$ (curves 2), 0.2 (curves 3,4), $\gamma=0$ (curves 3), 3.17 (curves 4)

of active centers $N \sim(2 \ldots 4) \cdot 10^{18} \mathrm{~cm}^{-3}$, optical wavelength $\sim 1.3 \cdot 10^{-6} \mathrm{~m}$. Fig. 1 illustrates the characteristic trajectories in the phase plane of variables $(|\rho|, n)$, which are calculated, following relation (2), for different types of interaction.

The law of conservation of the Bloch vector in the traditional formulation corresponds to the trajectory described by curve 1 in Fig. 1, a. Naturally, the trajectory has the shape of a circle, curve 2 has the same shape, corresponding only to taking into account phase relaxation. In this variant, however, the population difference in the negative part of the values has a smaller absolute value - the inversion is reset under the conditions of dipole deflation caused by the influence of spontaneous processes of interaction with the medium, it does not reach the minimum level $\left(n_{\min }=-1\right)$. To this extent, an incomplete reset of the inversion also leads to a decrease in the SR pulse power $\left(|\rho|^{2}<1\right)$. The influence of the phase effect associated with the dephasing of dipoles due to the difference in polarizabilities $(\beta \neq 0)$ leads to a deformation of the trajectory, describing the conservation law (curves 3 in both fragments of Fig. 1).

Pulse fronts should develop under the conditions of a different rate of change of inversion, so the generated "nonlinear" SR pulses become asymmetric. SR pulses should be characterized by an even greater difference in fronts in the case of the possibility of a dipole - dipole interaction (curves 4, Fig. 1). A relative increase in polarization, expressed by deformation of curves 4 , occurs due to the tuning of the resonance as the inversion is reset. The nonlinear dephasing of the elementary emitters forming the supercrystal, which caused asymmetry, can then occur under the simultaneous action of two self-modulating mechanisms with partial mutual compensation.

Using relation (5) enables accurate analytical calculation of the time sweep of the SR pulse taking into account phase relaxation and phase nonlinearities. From the relation for integral (5) follows the expression for $|\rho|^{2}$ :

$$
|\rho|^{2}=(1-n)\left\{1+n-\frac{2}{\kappa}+\frac{2}{\kappa} \beta(1-n)\left[\gamma-\frac{\beta}{3}\left(1+\gamma^{2}\right)(1-n)\right]\right\} .
$$

In view of (6), the inversion equation from (4) is solvable in the form of the relation:

$$
\begin{aligned}
& 2(\kappa-1) \frac{t-t_{0}}{T_{2}}=\ln (1-n)+\frac{3 \kappa-4}{2} \times \\
& \times \ln \left\{1+n-\frac{2}{\kappa}+\frac{2}{\kappa} \beta(1-n)\left[\gamma-\frac{\beta}{3}\left(1+\gamma^{2}\right)(1-n)\right]\right\}+ \\
& \frac{(3 \kappa-2+2 \beta \gamma)}{\sqrt{D}} \operatorname{Arth} \frac{\beta^{2}\left(1+\gamma^{2}\right)(1-n)+3(\kappa-2 \beta \gamma)}{\sqrt{D}}, \\
& D=(\kappa-2 \beta \gamma)^{2}+16 \beta^{2}(\kappa-1)\left(1+\gamma^{2}\right) / 3 .
\end{aligned}
$$



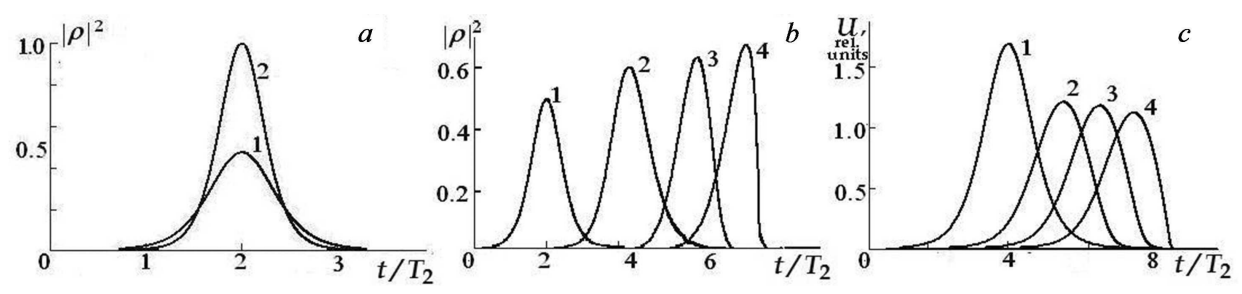

Fig. 2. Temporal dependences of the square of the polarization modulus $(a, b)$ and the normalized power of the light field $(c): \kappa=2.4(a), 2.2(b), 3.0(c), \beta=0$ (curve 1), 0.1 (2), 0.15 (3), 0.25 (4), $\gamma=3.17(b) ; \beta=0$ (1), $0.1(2)-(4), \gamma=1.58$ (2), 2.34 (3), 3.17 (4) (c); $T_{2}=1 \cdot 10^{-12} \mathrm{c}$

Here, we note that when neglecting the resonance phase nonlinearity, the integral (5) makes it possible to obtain solutions for the population and polarization in the form:

$$
\begin{aligned}
& n(t)=\operatorname{th}\left(\frac{t}{\tau}\right)+\frac{1}{\kappa} \operatorname{sh}\left(\frac{t}{\tau}\right) \exp \left(-\frac{t}{\tau}\right) \\
& |\rho|^{2}=\frac{(\kappa-1)^{2}}{\kappa^{2}} \operatorname{sh}^{2}\left(\frac{t}{\tau}\right), \quad \tau=\left(\frac{1}{T_{2}}-\frac{1}{\tau_{\mathrm{R}}}\right)^{-1} .
\end{aligned}
$$

Formally, dependence (8) is almost similar to the known [9] calculated for extended media and without taking into account phase relaxation. The ability to analytically take into account phase relaxation is provided by the homogeneous field approximation. Fig. 2, a shows, for comparison, the pulse profiles of resonant polarization with and without phase relaxation calculated in accordance with (8). It should be noted that the peak intensity of the light field pulses emitted under the conditions of relaxation mechanisms should decrease. This is directly related to the already noted factor of incomplete inversion dumping under the conditions of decay of the phased state of the dipole ensemble during the pulse. If to consider expression (6) relating $|\rho|$ and $n(t)$, we can establish that the minimum population difference $\mathrm{n}$, which is achieved when the SR pulse is illuminated after the inversion is reset during the transition of atoms to the ground state, increases, amounting to $n_{\min }^{\prime}=-1+2 / \kappa$.

Expression (4), taking into account the general case of interaction, allows a parametric calculation - the value $n$ can be used for this purpose as an argument that linearly grows within the limits determined by the integral (6).

Examples of computation of the temporal dependence of the variable $|\rho(t)|^{2}$ and the normalized intensity defined in scheme (4) using expressions $(6),(7)$ are shown in Fig. 2, $b$ and $c$. The increase in peak values $|\rho(t)|^{2}$ (Fig. 2, $b$ ) is also explained by the tuning of the gain resonance during the pulse development. However, this increase does not manifest itself in an increase in the peak pulse power (Fig. 2, c) due to its compensation by line broadening caused by the influence of absorption in quasi-resonant transitions (the presence of a polarizability defect $\alpha_{1}-\alpha_{2}$ ). The occurrence of pulse asymmetry is noted (curves 3,4 in Fig. 2, $b, c$ ), which increases in the case of the relative significance of the contributions of phase nonlinearity mechanisms - the existence of a difference in polarizabilities at the levels of the main transition (Fig. 2,b), and an increase in the dipole - dipole interaction degree (Fig. 2, c). It is assumed that the latter should occur due to an increase in the concentration of active centers $N$.

\section{Conclusions}

Some literature sources have predicted the possibility of self-excitation of generation in thin planar inverse layers (for example, [18]). The self-excitation mode, which can 
be realized in the form of superradiance under the considered conditions of achieving inverse population in a supercrystal formed by quantum dots, seems promising for use in obtaining pulsed lasing in laser devices. The gain element in compact devices can be a thin layer of a quasicrystal with a relatively high concentration of active centers while achieving a high inversion degree. The original calculated estimates of the time course of the SR process taking into account nonlinearities typical for the resonance response can be used to develop methods for obtaining and profiling optical pulses in the subpicosecond duration range in modern nanophotonic devices.

Acknowledgments. The work was carried out as part of the assignment 1.3.03 of the State Program of Scientific Research of the Republic of Belarus "Photonics, optoand microelectronics".

\section{References}

1. Krasnok A., Tymchenko M., Alú A. Nonlinear metasurfaces: A paradigm shift in nonlinear optics. Mater. Today, 2018, vol. 21, no. 1, pp. 8-21. doi: 10.1016/j.mattod.2017.06.007.

2. Bekenstein R., Pikovski I., Pichler H., Shahmoon E., Yelin S.F., Lukin M.D. Quantum metasurfaces with atom arrays. Nat. Phys., 2020, vol. 16, pp. 676-681. doi: 10.1038/s41567-020-0845-5.

3. Manzeli S., Ovchinnikov D., Pasquier D., Yazyev O.V., Kis A. 2D transition metal dichalcogenides. Nat. Rev. Mater., 2017, vol. 2, art. 17033, pp. 1-15. doi: 10.1038/natrevmats.2017.33.

4. Boneschanscher M.P., Evers W.H., Geuchies J.J., Altantzis T., Goris B., Rabouw F., van Rossum S.A.P., van der Zant H.S.J., Siebbeles L.D.A., Van Tendeloo G., Swart I., Hilhorst J., Petukhov A.V., Bals S., Vanmaekelbergh D. Long-range orientation and atomic attachment of nanocrystals in 2D honeycomb superlattices. Science, 2014, vol. 344, no. 6190 , pp. $1377-1380$. doi: $10.1126 /$ science. 1252642 .

5. Evers W.H., Goris B., Bals S., Casavola M., de Graaf J., van Roij R., Dijkstra M., Vanmaekelbergh D. Low-dimensional semiconductor superlattices formed by geometric control over nanocrystal attachment. Nano Lett., 2013, vol. 13, no. 6, pp. 2317-2323. doi: $10.1021 / \mathrm{nl} 303322 \mathrm{k}$.

6. Li L., Yu Y., Ye G.J., Ge Q., Ou X., Wu H., Feng D., Chen X.H., Zhang Yu. Black phosphorus field-effect transistors. Nat. Nanotechnol., 2014, vol. 9, pp. 372-377. doi: 10.1038/nnano.2014.35.

7. Liu W., Luo X., Bao Y., Liu Y.P., Ning G.-H., Abdelwahab I., Li L., Nai Ch.T., Hu Zh.G., Zhao D., Liu B., Quek S.Y., Loh K.P. A two-dimensional conjugated aromatic polymer via C-C coupling reaction. Nat. Chem., 2017, vol. 9, pp. 563-570. doi: 10.1038/nchem.2696.

8. Scuri G., Zhou Y., High A.A., Wild D.S., Shu Chi, De Greve K.,Jauregui L.A., Taniguchi T., Watanabe K., Kim Ph., Lukin M.D., Park H. Large excitonic reflectivity of monolayer $\mathrm{MoSe}_{2}$ encapsulated in hexagonal boron nitride. Phys. Rev. Lett., 2018, vol. 120, no. 3, art. 037402, pp. 1-6. doi: 10.1103/PhysRevLett.120.037402.

9. Andreev A.V., Emel'yanov V.I., Il'inskii Yu.A. Kooperativnye yavleniya v optike: Sverkhizluchenie. Bistabil'nost'. Fazovye perekhody [Cooperative Effects in Optics: Superradiation. Bistability. Phase Transitions]. Moscow, Nauka, 1988. 288 p. (In Russian)

10. Savel'ev A.V., Karachinskii L.Ya., Novikov I.I., Gordeev N.Yu., Seisyan R.P., Zegrya G.G. Formation of superradiance in quantum dot nanoheterostructures. Fiz. Tekh. Poluprovodn., 2008, vol. 42, no. 6, pp. 730-735. (In Russian)

11. Malikov R.F., Malyshev V.A. Optical bistability and hysteresis of a thin layer of resonant emitters: The mutual influence of inhomogeneous broadening of the absorption line and the Lorentz local field. Opt. Spectrosc., 2017, vol. 122, no. 6, pp. 955-963. doi: 10.1134/S0030400X17060121. 
12. Apanasevich P.A. Osnovy teorii vzaimodejstviya sveta s veshchestvom [Fundamentals of the Theory of Light-Matter Interaction]. Minsk, Nauka Tekh., 1977. 496 p. (In Russian)

13. Rupasov V.I., Yudson V.I. Boundary-value problems in nonlinear optics of resonant media] Sov. J. Quantum Electron., 1982, vol. 12, no. 11, pp. 1415-1419. doi: 10.1070/QE1982v012n11ABEH006090.

14. Zakharov S.M., Manykin E.A. Nonlinear interaction of light with a thin layer of surface resonant atoms. J. Exp. Theor. Phys., 1994, vol. 78, no. 4, pp. 566-571.

15. Ryzhov I.V., Malikov R.F., Malyshev A.V., Malyshev V.A. Nonlinear optical response of a two-dimensional quantum-dot supercrystal: Emerging multistability, periodic and aperiodic self-oscillations, chaos, and transient chaos. Phys. Rev. A, 2019, vol. 100, no. 3, art. 033820, pp. 1-15. doi: 10.1103/PhysRevA.100.033820.

16. Taranukhin V.D., Pogosbekyan M.Yu. Influence of optical Stark effect on the amplification of intense light pulses in resonance media. Quantum Electron., 1993, vol. 23, no. 8, pp. 713717. doi: 10.1070/QE1993v023n08ABEH003153.

17. Timoshchenko E.V., Yurevich V.A., Yurevich Yu.V. Resonance reflection of light by a thin layer of a dense nonlinear medium. Tech. Phys., 2013, vol. 58, no. 2, pp. 251-254. doi: $10.1134 / \mathrm{S} 1063784213020254$.

18. Petrov N.S., Zimin A.B. Laser generation in thin inverse layers. J. Appl. Spectrosc., 2010, vol. 77, no. 1, pp. 60-64. doi: 10.1007/s10812-010-9293-9.

Received

January 11, 2021

Timoshchenko Elena Valer'evna, PhD in Physical and Mathematical Sciences, Head of Department of Physics and Computer Technologies

Mogilev State A. Kuleshov University

ul. Kosmonavtov, 1, Mogilev, 212022 Republic of Belarus

E-mail: timoshchenko@msu.by

Yurevich Vladimir Antonovich, Doctor of Physical and Mathematical Sciences, Professor of Department of Technosphere Safety and General Physics

Belarusian State University of Food and Chemical Technologies pr. Shmidta, 3, Mogilev, 212027 Republic of Belarus

E-mail: va_yurevich@mail.ru

ОРИГИНАЛЬНАЯ СТАТЬЯ

УДК 535.23:621.378.825

doi: $10.26907 / 2541-7746.2021 .1 .21-30$

\title{
К решению задачи о нелинейном резонансном отклике квазидвумерного суперкристалла
}

\author{
Е.В. Тимощенко ${ }^{1}$, В.А. Юревич ${ }^{2}$ \\ ${ }^{1}$ Могилевский государственный университет имени А.А. Кулешова, \\ г. Могилев, 212022, Республика Беларусъ \\ ${ }^{2}$ Белорусский государственный университет пищевых и химических технологий, \\ г. Могилев, 212027, Республика Беларусъ
}

\section{Аннотация}

В работе приведены результаты исследования нелинейного оптического отклика двумерной решетки элементарных двухуровневых диполей, возникающий в условиях вынужденного излучения. Подобная резонансная реакция на световое поле реализуется 
в квазидвумерных полупроводниковых суперкристаллах (ПСК), формируемых квантовыми точками. Такие материалы являются актуальными в исследованиях и перспективными к использованию в нанофотонике.

Исследована динамика энергообмена среды ПСК, которая характеризуется относительно высокой концентрацией активных центров, и светового поля сверхизлучения. В приближении однородного поля решена задача теоретического описания коллективного эффекта сверхизлучения с учётом диполь-дипольного взаимодействия активных центров, типичного для ПСК и вызывающего нелинейное смещение резонансной частоты усиления (поглощения).

В расчётах использованы представления обобщённой двухуровневой схемы, позволяющие в квантовомеханических материальных уравнениях, решаемых совместно с уравнениями связи полей, учитывать также автомодуляционное спектральное уширение светового поля, обусловленное поглощением излучения в квазирезонансных переходах.

Определено соотношение, представляющее аналог закона сохранения полярного угла вектора Блоха для рассматриваемого более общего случая взаимодействия, в котором наряду с фазовой нелинейностью отклика учитывается скорость разброса активных диполей в пределах ширины спектральной линии (имеется в виду, конечность времени фазовой релаксации элементарных излучателей). Использование в этом случае формализма вектора Блоха позволяет получить аналитическое решение предложенной оригинальной модификации нелинейной системы уравнений для переменных отклика ПСК и рассчитать форму импульсов сверхизлучения. Расчётами предсказывается выраженная асимметрия излучаемых ПСК импульсов.

Ключевые слова: нелинейная динамика, суперкристаллы квантовых точек, резонансный отклик, диполь-дипольное взаимодействие, коллективные эффекты излучения, формализм вектора Блоха

Поступила в редакцию 11.01 .2021

Тимощенко Елена Валерьевна, кандидат физико-математических наук, заведующий кафедрой физики и компьютерных технологий

Могилевский государственный университет имени А.А. Кулешова ул. Космонавтов, д. 1, г. Могилев, 212022, Республика Беларусь

E-mail: timoshchenko@msu.by

Юревич Владимир Антонович, доктор физико-математических наук, профессор кафедры техносферной безопасности и общей физики

Белорусский государственный университет пищевых и химических технологий пр-т. Шмидта, д. 3, г. Могилев, 212027, Республика Беларусь

E-mail: va_yurevich@mail.ru

For citation: Timoshchenko E.V., Yurevich V.A. On solving the problem of quasi/ two-dimensional supercrystal nonlinear resonance response. Uchenye Zapiski Kazanskogo Universiteta. Seriya Fiziko-Matematicheskie Nauki, 2021, vol. 163, no. 1, pp. 21-30. doi: 10.26907/2541-7746.2021.1.21-30.

Для иитирования: Timoshchenko E.V., Yurevich V.A. On solving the problem of quasi-two-dimensional supercrystal nonlinear resonance response // Учен. зап. Казан. ун-та. Сер. Физ.-матем. науки. - 2021. - Т. 163, кн. 1. - С. 21-30. - doi: 10.26907/25417746.2021.1.21-30. 